\title{
Irrationality of the Roots of the Yablonskii-Vorob'ev Polynomials and Relations between Them
}

Pieter ROFFELSEN

Radboud Universiteit Nijmegen, IMAPP, FNWI, Heyendaalseweg 135, 6525 AJ Nijmegen, the Netherlands

E-mail: roffelse@science.ru.nl

Received November 13, 2010, in final form December 08, 2010; Published online December 14, 2010 doi:10.3842/SIGMA.2010.095

\begin{abstract}
We study the Yablonskii-Vorob'ev polynomials, which are special polynomials used to represent rational solutions of the second Painlevé equation. Divisibility properties of the coefficients of these polynomials, concerning powers of 4 , are obtained and we prove that the nonzero roots of the Yablonskii-Vorob'ev polynomials are irrational. Furthermore, relations between the roots of these polynomials for consecutive degree are found by considering power series expansions of rational solutions of the second Painlevé equation.
\end{abstract}

Key words: second Painlevé equation; rational solutions; power series expansion; irrational roots; Yablonskii-Vorob'ev polynomials

2010 Mathematics Subject Classification: 34M55

\section{Introduction}

In this paper we study the Yablonskii-Vorob'ev polynomials $Q_{n}$, with a special interest in their roots. These polynomials were derived by Yablonskii and Vorob'ev, while examining the hierarchy of rational solutions of the second Painlevé equation. The Yablonskii-Vorob'ev polynomials are defined by the differential-difference equation

$$
Q_{n+1} Q_{n-1}=z Q_{n}^{2}-4\left(Q_{n} Q_{n}^{\prime \prime}-\left(Q_{n}^{\prime}\right)^{2}\right),
$$

with $Q_{0}=1$ and $Q_{1}=z$. From the recurrence relation, it is clear that the functions $Q_{n}$ are rational, though it is far from obvious that they are polynomials, since in every iteration one divides by $Q_{n-1}$. The Yablonskii-Vorob'ev polynomials $Q_{n}$ are monic polynomials of degree $\frac{1}{2} n(n+1)$, with integer coefficients. The first few are given in Table 1 .

Yablonskii [1] and Vorob'ev [2] expressed the rational solutions of the second Painlevé equation,

$$
P_{\mathrm{II}}(\alpha): \quad w^{\prime \prime}(z)=2 w(z)^{3}+z w(z)+\alpha,
$$

with complex parameter $\alpha$, in terms of the Yablonskii-Vorob'ev polynomials, as summerized in the following theorem:

Theorem 1. $P_{\mathrm{II}}(\alpha)$ has a rational solution iff $\alpha=n \in \mathbb{Z}$. For $n \in \mathbb{Z}$ the rational solution is unique and if $n \geq 1$, then it is equal to

$$
w_{n}=\frac{Q_{n-1}^{\prime}}{Q_{n-1}}-\frac{Q_{n}^{\prime}}{Q_{n}} .
$$

The other rational solutions are given by $w_{0}=0$ and for $n \geq 1, w_{-n}=-w_{n}$. 
Table 1.

\begin{aligned} & \hline \multicolumn{1}{c}{ Yablonskii-Vorob'ev polynomials } \\ & \hline$Q_{2}= 4+z^{3} \\ & Q_{3}=-80+20 z^{3}+z^{6} \\ & Q_{4}= z\left(11200+60 z^{6}+z^{9}\right) \\ & Q_{5}=-6272000-3136000 z^{3}+78400 z^{6}+2800 z^{9}+140 z^{12}+z^{15} \\ & Q_{6}=-38635520000+19317760000 z^{3}+1448832000 z^{6}-17248000 z^{9}+627200 z^{12} \\ &+18480 z^{15}+280 z^{18}+z^{21} \\ & Q_{7}= z\left(-3093932441600000-49723914240000 z^{6}-828731904000 z^{9}+13039488000 z^{12}\right. \\ &\left.+62092800 z^{15}+5174400 z^{18}+75600 z^{21}+504 z^{24}+z^{27}\right) \\ & Q_{8}=-991048439693312000000-743286329769984000000 z^{3} \\ &+37164316488499200000 z^{6}+1769729356595200000 z^{9}+126696533483520000 z^{12} \\ &+407736096768000 z^{15}-6629855232000 z^{18}+124309785600 z^{21}+2018016000 z^{24} \\ &+32771200 z^{27}+240240 z^{30}+840 z^{33}+z^{36}\end{aligned}$

The rational solutions of $P_{\mathrm{II}}$ can also be determined, using the Bäcklund transformations, first given by Gambier [3], of the second Painlevé equation, by

$$
w_{n+1}=-w_{n}-\frac{2 n+1}{2 w_{n}^{2}+2 w_{n}^{\prime}+z}, \quad w_{-n}=-w_{n},
$$

with "seed solution" $w_{0}=0$; see also Lukashevich [4] and Noumi [5].

We note that the Yablonskii-Vorob'ev polynomials find many applications in physics. For instance, solutions of the Korteweg-de Vries equation (Airault, McKean and Moser [6]) and the Boussinesq equation (Clarkson [7]) can be expressed in terms of these polynomials. Clarkson and Mansfield [8] studied the structure of the roots of the Yablonskii-Vorob'ev polynomials $Q_{n}$ and observed that the roots, of each of these polynomials, form a highly regular triangular-like pattern, for $n \leq 7$, suggesting that they have interesting properties. This further motivates studying the zeros of the Yablonskii-Vorob'ev polynomials.

In Section 2 the divisibility of the coefficients of the Yablonskii-Vorob'ev polynomials by powers of 4 is examined. From the divisibility properties found, we conclude that nonzero roots of the Yablonskii-Vorob'ev polynomials are irrational. In Section 3 we study power series expansions of (functions related to) the rational solution $w_{n}$ of $P_{\mathrm{II}}(n)$, around poles of $w_{n}$. This leads to relations between the roots of $Q_{n-1}$ and $Q_{n}$. These relations suggest deeper connections between the zeros of $Q_{n-1}$ and $Q_{n}$. Similarly, we look at power series expansions of (functions related to) the rational solution $w_{n}$ of $P_{\mathrm{II}}(n)$ around 0 , in Section 4. We obtain polynomial expressions in $n$, with rational coefficients, for sums of fixed negative powers of the nonzero roots of $Q_{n}$.

\section{Nonzero roots are irrational}

The Yablonskii-Vorob'ev polynomials $Q_{n}$ are monic polynomials of degree $\frac{1}{2} n(n+1)$, and Taneda [9] proved:

- if $n \equiv 1(\bmod 3)$, then $\frac{Q_{n}}{z} \in \mathbb{Z}\left[z^{3}\right]$;

- if $n \not \equiv 1(\bmod 3)$, then $Q_{n} \in \mathbb{Z}\left[z^{3}\right]$.

Therefore, we have

$$
Q_{n}=z^{\frac{1}{2} n(n+1)}+a_{1}^{n} z^{\frac{1}{2} n(n+1)-3}+a_{2}^{n} z^{\frac{1}{2} n(n+1)-6}+\cdots+a_{\left[\frac{1}{6} n(n+1)\right.}^{n} z^{\frac{1}{2} n(n+1)-3\left[\frac{1}{6} n(n+1)\right]},
$$

for certain $a_{s}^{n} \in \mathbb{Z}$, with convention $a_{0}^{n}=1$, where $[\cdot]$ denotes the floor function. 
Lemma 1. For every $0 \leq m \leq\left[\frac{1}{6} n(n+1)\right]$, we have $4^{m} \mid a_{m}^{n}$.

Proof. We proceed by proving the following statement, by induction, for all $M \in \mathbb{N}$ :

For every $1 \leq m \leq M$, for all $n \in \mathbb{N}$, whenever $m \leq\left[\frac{1}{6} n(n+1)\right]$, we have $4^{m} \mid a_{m}^{n}$, and

$$
4^{M}\left|a_{M+1}^{n}, \quad 4^{M}\right| a_{M+2}^{n}, \quad \ldots, \quad 4^{M} \mid a_{\left[\frac{1}{6} n(n+1)\right]}^{n} .
$$

Observe that the case $M=0$ is trivial. Now suppose the statement is true for $M \in \mathbb{N}$. Then there are $b_{s}^{n} \in \mathbb{Z}$, such that for every $n \in \mathbb{N}$,

$$
Q_{n}=z^{\frac{1}{2} n(n+1)}+4 b_{1}^{n} z^{\frac{1}{2} n(n+1)-3}+4^{2} b_{2}^{n} z^{\frac{1}{2} n(n+1)-6}+\cdots+4^{M} b_{M}^{n} z^{\frac{1}{2} n(n+1)-3 M}+4^{M} P_{n},
$$

where $P_{n} \in \mathbb{Z}[z]$ is zero or has degree less or equal to $\frac{1}{2} n(n+1)-3(M+1)$, and if $m>\left[\frac{1}{6} n(n+1)\right]$, then $b_{m}^{n}=0$.

To complete the induction, we need to show that for every $n \in \mathbb{N}, 4 \mid P_{n}$. We prove this by induction with respect to $n$. Observe that $P_{0}=0$ and $P_{1}=0$, therefore, indeed $4 \mid P_{0}$ and $4 \mid P_{1}$. Assume $4 \mid P_{n-1}$ and $4 \mid P_{n}$. Then $4^{M} P_{n} \equiv 0\left(\bmod 4^{M+1}\right)$, therefore, modulo $4^{M+1}$, we have:

$$
\begin{aligned}
& z^{\max (0, n(n+1)-3 M+1)}\left|z Q_{n}^{2}, \quad z^{\max (0, n(n+1)-3 M+1)}\right| 4 Q_{n} Q_{n}^{\prime \prime}, \\
& z^{\max (0, n(n+1)-3 M+1)} \mid 4\left(Q_{n}^{\prime}\right)^{2} .
\end{aligned}
$$

By the definition of $Q_{n+1}(1)$,

$$
Q_{n+1} Q_{n-1}=z Q_{n}^{2}-4\left(Q_{n} Q_{n}^{\prime \prime}-\left(Q_{n}^{\prime}\right)^{2}\right),
$$

SO

$$
z^{\max (0, n(n+1)-3 M+1)} \mid Q_{n+1} Q_{n-1} \quad\left(\bmod 4^{M+1}\right) .
$$

Let us consider $Q_{n+1} Q_{n-1}$. Since $4 \mid P_{n-1}$, we have

$$
4^{M} P_{n-1} \equiv 0 \quad\left(\bmod 4^{M+1}\right),
$$

therefore, modulo $4^{M+1}$,

$$
\begin{aligned}
Q_{n+1} Q_{n-1} \equiv & Q_{n+1} z^{\frac{1}{2} n(n-1)}+Q_{n+1}\left(4 b_{1}^{n-1} z^{\frac{1}{2} n(n-1)-3}\right. \\
& \left.+4^{2} b_{2}^{n-1} z^{\frac{1}{2} n(n-1)-6}+\cdots+4^{M} b_{M}^{n-1} z^{\frac{1}{2} n(n-1)-3 M}\right) .
\end{aligned}
$$

Since

$$
\begin{aligned}
Q_{n+1}= & z^{\frac{1}{2}(n+1)(n+2)}+4 b_{1}^{n+1} z^{\frac{1}{2}(n+1)(n+2)-3} \\
& +4^{2} b_{2}^{n+1} z^{\frac{1}{2}(n+1)(n+2)-6}+\cdots+4^{M} b_{M}^{n+1} z^{\frac{1}{2}(n+1)(n+2)-3 M}+4^{M} P_{n+1},
\end{aligned}
$$

we have, modulo $4^{M+1}$,

$$
\begin{aligned}
& z^{\max (0, n(n+1)-3 M+1)} \mid Q_{n+1}\left(4 b_{1}^{n-1} z^{\frac{1}{2} n(n-1)-3}+4^{2} b_{2}^{n-1} z^{\frac{1}{2} n(n-1)-6}\right. \\
& \left.+\cdots+4^{M} b_{M}^{n-1} z^{\frac{1}{2} n(n-1)-3 M}\right) .
\end{aligned}
$$

Hence, by (3) and (4),

$$
z^{\max (0, n(n+1)-3 M+1)} \mid Q_{n+1} z^{\frac{1}{2} n(n-1)} \quad\left(\bmod 4^{M+1}\right),
$$


which implies

$$
z^{\max \left(0, \frac{1}{2}(n+1)(n+2)-3 M\right)} \mid Q_{n+1} \quad\left(\bmod 4^{M+1}\right) .
$$

Since

$$
\begin{aligned}
Q_{n+1}= & z^{\frac{1}{2}(n+1)(n+2)}+4 b_{1}^{n+1} z^{\frac{1}{2}(n+1)(n+2)-3} \\
& +4^{2} b_{2}^{n+1} z^{\frac{1}{2}(n+1)(n+2)-6}+\cdots+4^{M} b_{M}^{n+1} z^{\frac{1}{2}(n+1)(n+2)-3 M}+4^{M} P_{n+1},
\end{aligned}
$$

we have, therefore, $4 \mid P_{n+1}$. Hence, by induction, for all $n \in \mathbb{N}, 4 \mid P_{n}$.

The lemma follows by induction on $M$.

Let us denote the coefficient of the lowest degree term in $Q_{n}$ by

$$
x_{n}:=a_{\left[\frac{1}{6} n(n+1)\right]}^{n},
$$

i.e. $x_{n}$ is the constant coefficient in $Q_{n}$ if $n \not \equiv 1(\bmod 3)$, and $x_{n}$ is the coefficient of $z$ in $Q_{n}$ if $n \equiv 1(\bmod 3)$. Fukutani, Okamoto, and Umemura [10] proved that the roots of the YablonskiiVorob'ev polynomials are simple, hence $x_{n}$ is nonzero. Let $p_{n}$ be the multiplicity of 2 in the prime factorization of $x_{n}$. As a consequence of Lemma 1, we obtain that $p_{n} \geq 2\left[\frac{1}{6} n(n+1)\right]$. We prove

$$
p_{n}=\left[\frac{1}{3} n(n+1)\right] .
$$

Observe that $x_{n}=Q_{n}(0)$ if $n \not \equiv 1(\bmod 3)$, and $x_{n}=Q_{n}^{\prime}(0)$ if $n \equiv 1(\bmod 3)$. Fukutani, Okamoto, and Umemura [10] derived the following identity for the Yablonskii-Vorob'ev polynomials:

$$
Q_{n+1}^{\prime} Q_{n-1}-Q_{n+1} Q_{n-1}^{\prime}=(2 n+1) Q_{n}^{2}
$$

Using this identity at 0 , we obtain

$$
x_{n+1} x_{n-1}=\left\{\begin{array}{lll}
(2 n+1) x_{n}^{2} & \text { if } n \equiv 0 & (\bmod 3), \\
-(2 n+1) x_{n}^{2} & \text { if } n \equiv 2 & (\bmod 3) .
\end{array}\right.
$$

By evaluating equation (1) at 0 ,

$$
x_{n+1} x_{n-1}=4 x_{n}^{2}, \quad \text { if } n \equiv 1 \quad(\bmod 3) .
$$

Therefore, we have the following recursion for $\left(x_{n}\right)_{n}$ :

$$
\begin{array}{ll}
x_{0}=1, \quad & x_{1}=1 \quad \text { and } \\
x_{n+1} x_{n-1}=\left\{\begin{array}{lll}
(2 n+1) x_{n}^{2} & \text { if } n \equiv 0 & (\bmod 3), \\
4 x_{n}^{2} & \text { if } n \equiv 1 & (\bmod 3), \\
-(2 n+1) x_{n}^{2} & \text { if } n \equiv 2 & (\bmod 3) .
\end{array}\right.
\end{array}
$$

So, we obtain the following recursion for $\left(p_{n}\right)_{n}$ :

$$
\begin{aligned}
& p_{0}=0, \quad p_{1}=0 \quad \text { and } \\
& p_{n+1}=\left\{\begin{array}{lll}
2 p_{n}-p_{n-1} & \text { if } n \not \equiv 1 \\
2+2 p_{n}-p_{n-1} & \text { if } n \equiv 1 & (\bmod 3),
\end{array}\right.
\end{aligned}
$$

Using this recursion, the formula $p_{n}=\left[\frac{1}{3} n(n+1)\right]$, can be proven directly, by induction. 
Remark 1. Kaneko and Ochiai [11] found an explicit expression for the coefficients $x_{n}$. But deriving the formula $p_{n}=\left[\frac{1}{3} n(n+1)\right]$ directly from this expression seems to be a difficult task.

Theorem 2. The nonzero roots of the Yablonskii-Vorob'ev polynomials are irrational.

Proof. Let $n \not \equiv 1(\bmod 3)$. Suppose $x$ is a rational root of $Q_{n}$. Since $Q_{n} \in \mathbb{Z}[z]$ is monic, by Gauss's lemma, $x \in \mathbb{Z}$. By Lemma 1 ,

$$
Q_{n} \equiv z^{\frac{1}{2} n(n+1)} \quad(\bmod 4)
$$

so $x$ is even. Let $y:=\frac{x}{2}$, then, by equation (2),

$$
0=(2 y)^{\frac{1}{2} n(n+1)}+a_{1}^{n}(2 y)^{\frac{1}{2} n(n+1)-3}+a_{2}^{n}(2 y)^{\frac{1}{2} n(n+1)-6}+\cdots+a_{\frac{1}{6} n(n+1)-1}^{n}(2 y)^{3}+a_{\frac{1}{6} n(n+1)}^{n} .
$$

By Lemma 1, for every $m \leq \frac{1}{6} n(n+1)$, we have $4^{m} \mid a_{m}^{n}$. Hence

$$
\begin{aligned}
& 2^{\frac{1}{2} n(n+1)}\left|(2 y)^{\frac{1}{2} n(n+1)}, \quad 2^{\frac{1}{2} n(n+1)-1}\right| a_{1}^{n}(2 y)^{\frac{1}{2} n(n+1)-3}, \\
& 2^{\frac{1}{2} n(n+1)-2}\left|a_{2}^{n}(2 y)^{\frac{1}{2} n(n+1)-6}, \quad \ldots, \quad 2^{\frac{1}{2} n(n+1)-\frac{1}{6} n(n+1)+1}\right| a_{\frac{1}{6} n(n+1)-1}(2 y)^{3} .
\end{aligned}
$$

So

$$
2^{\frac{1}{3} n(n+1)+1} \mid a_{\frac{1}{6} n(n+1)}^{n}=x_{n},
$$

which implies

$$
p_{n} \geq \frac{1}{3} n(n+1)+1
$$

But $p_{n}=\frac{1}{3} n(n+1)$, a contradiction, hence roots of $Q_{n}$ are irrational.

If $n \equiv 1(\bmod 3)$, we can apply the same reasoning to $\frac{Q_{n}}{z}$, and show that roots of $\frac{Q_{n}}{z}$ are irrational. Therefore, nonzero roots of $Q_{n}$ are irrational.

This result raises the question whether the Yablonskii-Vorob'ev polynomials, excluding the trivial factor $z$ in case $n \equiv 1(\bmod 3)$, are irreducible in $\mathbb{Q}[z]$. Kametaka [12] showed that for $n \leq 23$, the Yablonskii-Vorob'ev polynomials $Q_{n}$ are indeed irreducible.

\section{Relations between roots of the Yablonskii-Vorob'ev polynomials}

By Theorem 1, for $n \geq 1$, the unique rational solution of $P_{\mathrm{II}}(n)$ is given by

$$
w_{n}=\frac{Q_{n-1}^{\prime}}{Q_{n-1}}-\frac{Q_{n}^{\prime}}{Q_{n}} .
$$

Fukutani, Okamoto, and Umemura [10] proved that the roots of the Yablonskii-Vorob'ev polynomials are simple, hence

$$
w_{n}=\sum_{k=1}^{\frac{1}{2} n(n-1)} \frac{1}{z-z_{n-1, k}}-\sum_{k=1}^{\frac{1}{2} n(n+1)} \frac{1}{z-z_{n, k}},
$$

where the $z_{m, k}$ are the roots of $Q_{m}$. From equation (5) and the fact that $w_{n}$ is the rational solution of $P_{\mathrm{II}}(n)$, we obtain relations between the zeros of $Q_{n-1}$ and $Q_{n}$. 
Theorem 3. For $1 \leq j \leq \frac{1}{2} n(n-1)$ :

$$
\begin{aligned}
& \sum_{k=1, k \neq j}^{\frac{1}{2} n(n-1)} \frac{1}{z_{n-1, j}-z_{n-1, k}}-\sum_{k=1}^{\frac{1}{2} n(n+1)} \frac{1}{z_{n-1, j}-z_{n, k}}=0, \\
& \sum_{k=1, k \neq j}^{\frac{1}{2} n(n-1)} \frac{1}{\left(z_{n-1, j}-z_{n-1, k}\right)^{2}}-\sum_{k=1}^{\frac{1}{2} n(n+1)} \frac{1}{\left(z_{n-1, j}-z_{n, k}\right)^{2}}=\frac{z_{n-1, j}}{6}, \\
& \sum_{k=1, k \neq j}^{\frac{1}{2} n(n-1)} \frac{1}{\left(z_{n-1, j}-z_{n-1, k}\right)^{3}}-\sum_{k=1}^{\frac{1}{2} n(n+1)} \frac{1}{\left(z_{n-1, j}-z_{n, k}\right)^{3}}=-\frac{n+1}{4}, \\
& \sum_{k=1, k \neq j}^{\frac{1}{2} n(n-1)} \frac{1}{\left(z_{n-1, j}-z_{n-1, k}\right)^{5}}-\sum_{k=1}^{\frac{1}{2} n(n+1)} \frac{1}{\left(z_{n-1, j}-z_{n, k}\right)^{5}}=z_{n-1, j}\left(\frac{n+1}{24}-\frac{1}{36}\right) .
\end{aligned}
$$

For $1 \leq j \leq \frac{1}{2} n(n+1)$ :

$$
\begin{aligned}
& \sum_{k=1}^{\frac{1}{2} n(n-1)} \frac{1}{z_{n, j}-z_{n-1, k}}-\sum_{k=1, k \neq j}^{\frac{1}{2} n(n+1)} \frac{1}{z_{n, j}-z_{n, k}}=0, \\
& \sum_{k=1}^{\frac{1}{2} n(n-1)} \frac{1}{\left(z_{n, j}-z_{n-1, k}\right)^{2}}-\sum_{k=1, k \neq j}^{\frac{1}{2} n(n+1)} \frac{1}{\left(z_{n, j}-z_{n, k}\right)^{2}}=-\frac{z_{n, j}}{6}, \\
& \sum_{k=1}^{\frac{1}{2} n(n-1)} \frac{1}{\left(z_{n, j}-z_{n-1, k}\right)^{3}}-\sum_{k=1, k \neq j}^{\frac{1}{2} n(n+1)} \frac{1}{\left(z_{n, j}-z_{n, k}\right)^{3}}=-\frac{n-1}{4}, \\
& \sum_{k=1}^{\frac{1}{2} n(n-1)} \frac{1}{\left(z_{n, j}-z_{n-1, k}\right)^{5}}-\sum_{k=1, k \neq j}^{\frac{1}{2} n(n+1)} \frac{1}{\left(z_{n, j}-z_{n, k}\right)^{5}}=z_{n, j}\left(\frac{n-1}{24}+\frac{1}{36}\right) .
\end{aligned}
$$

Proof. Let $1 \leq j \leq \frac{1}{2} n(n-1)$ and define $\omega:=z_{n-1, j}$ and $u:=w_{n}-\frac{1}{z-\omega}$. Since $\operatorname{gcd}\left(Q_{n-1}, Q_{n}\right)=1$, see Fukutani, Okamoto, and Umemura [10], equation (5) shows that $u$ is holomorphic in a neighbourhood of $\omega$. Hence $u$ has a power series expansion, say

$$
\sum_{m=0}^{\infty} a_{m}(z-\omega)^{m},
$$

which converges in an open disc centered at $\omega$.

Since $w_{n}$ is a solution of $P_{\mathrm{II}}(n), u$ satisfies

$$
\begin{aligned}
(z-\omega)^{2} u^{\prime \prime}= & 6 u+6(z-\omega) u^{2}+2(z-\omega)^{2} u^{3}+(n+1)(z-\omega)^{2}+\omega(z-\omega) \\
& +(z-\omega)^{3} u+\omega(z-\omega)^{2} u
\end{aligned}
$$

Hence we have the following identity in an open disc centered at $\omega$ :

$$
\begin{aligned}
& \sum_{m=2}^{\infty}(m-1) m a_{m}(z-\omega)^{m}=6 \sum_{m=0}^{\infty} a_{m}(z-\omega)^{m}+6(z-\omega)\left(\sum_{m=0}^{\infty} a_{m}(z-\omega)^{m}\right)^{2} \\
& \quad+2(z-\omega)^{2}\left(\sum_{m=0}^{\infty} a_{m}(z-\omega)^{m}\right)^{3}+(n+1)(z-\omega)^{2}+\omega(z-\omega)
\end{aligned}
$$




$$
+(z-\omega)^{3} \sum_{m=0}^{\infty} a_{m}(z-\omega)^{m}+\omega(z-\omega)^{2} \sum_{m=0}^{\infty} a_{m}(z-\omega)^{m} .
$$

By considering coefficients of $(z-\omega)^{n}, n=0,1,2,4$, it is easy to deduce that $a_{0}=0, a_{1}=-\frac{\omega}{6}$, $a_{2}=-\frac{n+1}{4}$ and $a_{4}=\omega\left(\frac{n+1}{24}-\frac{1}{36}\right)$. Note that $a_{3}$ does not follow from considering coefficients of $(z-\omega)^{3}$.

By Taylor's theorem and equation (5),

$$
a_{m}=\frac{u^{(m)}\left(z_{n-1, j}\right)}{m !}=(-1)^{m}\left(\sum_{k=1, k \neq j}^{\frac{1}{2} n(n-1)} \frac{1}{\left(z_{n-1, j}-z_{n-1, k}\right)^{m+1}}-\sum_{k=1}^{\frac{1}{2} n(n+1)} \frac{1}{\left(z_{n-1, j}-z_{n, k}\right)^{m+1}}\right) .
$$

The first half of the theorem follows, the second half is proved analogously.

Note that countably many nontrivial relations can be found between the $a_{m}$ in the above proof, by considering the coefficient of $(z-\omega)^{n}$, for $n \in \mathbb{N}$.

In Kudryashov and Demina [13] similar relations for the roots of $Q_{n}$ are obtained using the Korteweg-de Vries equation. In particular, the following results are presented in [13] for $1 \leq j \leq \frac{1}{2} n(n+1)$ :

$$
\begin{aligned}
& \sum_{k=1, k \neq j}^{\frac{1}{2} n(n+1)} \frac{1}{\left(z_{n, j}-z_{n, k}\right)^{2}}=-\frac{z_{n, j}}{12}, \quad \sum_{k=1, k \neq j}^{\frac{1}{2} n(n+1)} \frac{1}{\left(z_{n, j}-z_{n, k}\right)^{3}}=0, \\
& \sum_{k=1, k \neq j}^{\frac{1}{2} n(n+1)} \frac{1}{\left(z_{n, j}-z_{n, k}\right)^{5}}=-\frac{z_{n, j}}{144} .
\end{aligned}
$$

From these relations and Theorem 3, we obtain the following corollary:

Corollary 1. For $1 \leq j \leq \frac{1}{2} n(n-1)$ :

$$
\begin{aligned}
& \sum_{k=1}^{\frac{1}{2} n(n+1)} \frac{1}{\left(z_{n-1, j}-z_{n, k}\right)^{2}}=-\frac{z_{n-1, j}}{4}, \quad \sum_{k=1}^{\frac{1}{2} n(n+1)} \frac{1}{\left(z_{n-1, j}-z_{n, k}\right)^{3}}=\frac{n+1}{4}, \\
& \sum_{k=1}^{\frac{1}{2} n(n+1)} \frac{1}{\left(z_{n-1, j}-z_{n, k}\right)^{5}}=-z_{n-1, j}\left(\frac{n+1}{24}-\frac{1}{48}\right) .
\end{aligned}
$$

For $1 \leq j \leq \frac{1}{2} n(n+1)$ :

$$
\begin{aligned}
& \sum_{k=1}^{\frac{1}{2} n(n-1)} \frac{1}{\left(z_{n, j}-z_{n-1, k}\right)^{2}}=-\frac{z_{n, j}}{4}, \quad \sum_{k=1}^{\frac{1}{2} n(n-1)} \frac{1}{\left(z_{n, j}-z_{n-1, k}\right)^{3}}=-\frac{n-1}{4}, \\
& \sum_{k=1}^{\frac{1}{2} n(n-1)} \frac{1}{\left(z_{n, j}-z_{n-1, k}\right)^{5}}=z_{n, j}\left(\frac{n-1}{24}+\frac{1}{48}\right) .
\end{aligned}
$$

In Theorem 3, we have obtained 4 times $\frac{1}{2} n(n-1)$ plus 4 times $\frac{1}{2} n(n+1)$ equations satisfied by the $\frac{1}{2} n(n+1)$ roots of $Q_{n}$, suggesting that these equations can be used to determine the roots of the polynomials $Q_{n}$ recursively. If so, then these equations may be of use to derive properties of the roots of the Yablonskii-Vorob'ev polynomials. We shall not pursue this issue further here. 


\section{Sums of negative powers of roots}

In Section 2, the rational solutions $w_{n}$ of $P_{\mathrm{II}}(n)$ were studied around roots of the YablonskiiVorob'ev polynomials. In this section, we consider $w_{n}$ at 0 .

Let $n \equiv 0(\bmod 3)$, then 0 is not a root of $Q_{n-1}$ or $Q_{n}$. Therefore, by equation $(5), w_{n}$ is holomorphic in a neighbourhood of 0 . So $w_{n}$ has a power series expansion, say

$$
\sum_{m=0}^{\infty} a_{m} z^{m}
$$

which converges on an open disc centered at 0 .

By Taylor's theorem and equation (5), we have

$$
a_{m}=-\left(\sum_{k=1}^{\frac{1}{2} n(n-1)} \frac{1}{z_{n-1, k}^{m+1}}-\sum_{k=1}^{\frac{1}{2} n(n+1)} \frac{1}{z_{n, k}^{m+1}}\right) .
$$

Let $\omega:=e^{\frac{2 \pi i}{3}}$. Since $n \equiv 0(\bmod 3), Q_{n} \in \mathbb{Z}\left[z^{3}\right]$. Therefore, the roots of $Q_{n}$ are invariant under multiplication by $\omega$. Hence

$$
\sum_{k=1}^{\frac{1}{2} n(n+1)} \frac{1}{z_{n, k}^{m+1}}=\sum_{k=1}^{\frac{1}{2} n(n+1)} \frac{1}{\left(\omega z_{n, k}\right)^{m+1}}=\frac{1}{\omega^{m+1}} \sum_{k=1}^{\frac{1}{2} n(n+1)} \frac{1}{z_{n, k}^{m+1}}
$$

therefore, if $m \not \equiv 2(\bmod 3)$,

$$
\sum_{k=1}^{\frac{1}{2} n(n+1)} \frac{1}{z_{n, k}^{m+1}}=0
$$

By the same reason, if $m \not \equiv 2(\bmod 3)$,

$$
\sum_{k=1}^{\frac{1}{2} n(n-1)} \frac{1}{z_{n-1, k}^{m+1}}=0
$$

So $a_{m}=0$, if $m \not \equiv 2(\bmod 3)$, and in an open disc centered at 0 ,

$$
w_{n}(z)=\sum_{m=0}^{\infty} a_{3 m+2} z^{3 m+2} .
$$

Since $w_{n}$ is a solution of $P_{\mathrm{II}}(n)$, we have the following identity in an open disc centered at 0 :

$$
\sum_{m=0}^{\infty}(3 m+1)(3 m+2) a_{3 m+2} z^{3 m}=2\left(\sum_{m=0}^{\infty} a_{3 m+2} z^{3 m+2}\right)^{3}+\sum_{m=0}^{\infty} a_{3 m+2} z^{3 m+3}+n .
$$

Comparing coefficients gives $a_{2}=\frac{1}{2} n, a_{5}=\frac{1}{40} n$ and $a_{8}=\frac{1}{2240} n+\frac{1}{224} n^{3}$. We have obtained the following relations for $n \equiv 0(\bmod 3)$ :

$$
\sum_{k=1}^{\frac{1}{2} n(n-1)} \frac{1}{z_{n-1, k}^{3}}-\sum_{k=1}^{\frac{1}{2} n(n+1)} \frac{1}{z_{n, k}^{3}}=-\frac{n}{2}, \quad \sum_{k=1}^{\frac{1}{2} n(n-1)} \frac{1}{z_{n-1, k}^{6}}-\sum_{k=1}^{\frac{1}{2} n(n+1)} \frac{1}{z_{n, k}^{6}}=-\frac{n}{40},
$$




$$
\sum_{k=1}^{\frac{1}{2} n(n-1)} \frac{1}{z_{n-1, k}^{9}}-\sum_{k=1}^{\frac{1}{2} n(n+1)} \frac{1}{z_{n, k}^{9}}=-\frac{1}{2240} n-\frac{1}{224} n^{3} .
$$

If $n \equiv 1(\bmod 3)$, then $u:=w_{n}+\frac{1}{z}$ is holomorphic at 0 and satisfies

$$
z^{2} u^{\prime \prime}=6 u-6 z u^{2}+2 z^{2} u^{3}+z^{3} u+(n-1) z^{2} .
$$

By considering the power series expansion of $u=w_{n}+\frac{1}{z}$ around 0 , the following relations are found:

$$
\begin{aligned}
& \sum_{k=1}^{\frac{1}{2} n(n-1)} \frac{1}{z_{n-1, k}^{3}}-\sum_{k=1, z_{n, k} \neq 0}^{\frac{1}{2} n(n+1)} \frac{1}{z_{n, k}^{3}}=\frac{1}{4}(n-1), \\
& \sum_{k=1}^{\frac{1}{2} n(n-1)} \frac{1}{z_{n-1, k}^{6}}-\sum_{k=1, z_{n, k} \neq 0}^{\frac{1}{2} n(n+1)} \frac{1}{z_{n, k}^{6}}=\frac{1}{56}(n-1)+\frac{3}{112}(n-1)^{2}, \\
& \sum_{k=1}^{\frac{1}{2} n(n-1)} \frac{1}{z_{n-1, k}^{9}}-\sum_{k=1, z_{n, k} \neq 0}^{\frac{1}{2} n(n+1)} \frac{1}{z_{n, k}^{9}}=\frac{1}{2800}(n-1)+\frac{9}{5600}(n-1)^{2}+\frac{1}{448}(n-1)^{3} .
\end{aligned}
$$

If $n \equiv 2(\bmod 3)$, then $u:=w_{n}-\frac{1}{z}$ is holomorphic at 0 and satisfies

$$
z^{2} u^{\prime \prime}=6 u-6 z u^{2}+2 z^{2} u^{3}+z^{3} u+(n+1) z^{2} .
$$

By considering the power series expansion of $u=w_{n}-\frac{1}{z}$ around 0 , the following relations are found:

$$
\begin{aligned}
& \sum_{k=1, z_{n-1, k} \neq 0}^{\frac{1}{2} n(n-1)} \frac{1}{z_{n-1, k}^{3}}-\sum_{k=1}^{\frac{1}{2} n(n+1)} \frac{1}{z_{n, k}^{3}}=\frac{1}{4}(n+1), \\
& \sum_{k=1, z_{n-1, k} \neq 0}^{\frac{1}{2} n(n-1)} \frac{1}{z_{n-1, k}^{6}}-\sum_{k=1}^{\frac{1}{2} n(n+1)} \frac{1}{z_{n, k}^{6}}=\frac{1}{56}(n+1)-\frac{3}{112}(n+1)^{2}, \\
& \sum_{k=1, z_{n-1, k} \neq 0}^{\frac{1}{2} n(n-1)} \frac{1}{z_{n-1, k}^{9}}-\sum_{k=1}^{\frac{1}{2} n(n+1)} \frac{1}{z_{n, k}^{9}}=\frac{1}{2800}(n+1)-\frac{9}{5600}(n+1)^{2}+\frac{1}{448}(n+1)^{3} .
\end{aligned}
$$

Remark 2. Considering higher order coefficients, we see that for every threefold $m \geq 3$, polynomial expressions in $n$, with rational coefficients, depending on $n(\bmod 3)$, exist for

$$
\sum_{k=1, z_{n-1, k} \neq 0}^{\frac{1}{2} n(n-1)} \frac{1}{z_{n-1, k}^{m}}-\sum_{k=1, z_{n, k} \neq 0}^{\frac{1}{2} n(n+1)} \frac{1}{z_{n, k}^{m}} .
$$

As a corollary of these relations, by induction, we obtain:

$$
\sum_{k=1, z_{n, k} \neq 0}^{\frac{1}{2} n(n+1)} \frac{1}{z_{n, k}^{3}}=\left\{\begin{array}{lll}
\frac{n}{4} & \text { if } n \equiv 0 & (\bmod 3), \\
0 & \text { if } n \equiv 1 & (\bmod 3), \\
-\frac{n+1}{4} & \text { if } n \equiv 2 & (\bmod 3),
\end{array}\right.
$$




$$
\begin{aligned}
& \sum_{k=1, z_{n, k} \neq 0}^{\frac{1}{2} n(n+1)} \frac{1}{z_{n, k}^{6}}= \begin{cases}\frac{1}{40} n^{2}+\frac{1}{80} n & \text { if } n \equiv 0 \quad(\bmod 3), \\
-\frac{1}{560} n^{2}-\frac{1}{560} n+\frac{1}{280} & \text { if } n \equiv 1 \quad(\bmod 3), \\
\frac{1}{40} n^{2}+\frac{3}{80} n+\frac{1}{80} & \text { if } n \equiv 2 \quad(\bmod 3),\end{cases} \\
& \sum_{k=1, z_{n, k} \neq 0}^{\frac{1}{2} n(n+1)} \frac{1}{z_{n, k}^{9}}= \begin{cases}\frac{n+7 n^{2}+10 n^{3}}{4480} & \text { if } n \equiv 0 \quad(\bmod 3), \\
\frac{2-n-n^{2}}{22400} & \text { if } n \equiv 1 \quad(\bmod 3), \\
\frac{-20-85 n-115 n^{2}-50 n^{3}}{22400} & \text { if } n \equiv 2 \quad(\bmod 3) .\end{cases}
\end{aligned}
$$

By Remark 2, for every threefold $m \geq 3$, polynomial expressions in $n$, with rational coefficients, depending on $n(\bmod 3)$, exist for

$$
\sum_{k=1, z_{n, k} \neq 0}^{\frac{1}{2} n(n+1)} \frac{1}{z_{n, k}^{m}} .
$$

If $m \not \equiv 0(\bmod 3)$, see equation $(6)$, then

$$
\sum_{k=1, z_{n, k} \neq 0}^{\frac{1}{2} n(n+1)} \frac{1}{z_{n, k}^{m}}=0 .
$$

So, for all $n, m \in \mathbb{N}$,

$$
\sum_{k=1, z_{n, k} \neq 0}^{\frac{1}{2} n(n+1)} \frac{1}{z_{n, k}^{m}} \in \mathbb{Q},
$$

even though the nonzero roots of the Yablonskii-Vorob'ev polynomials are irrational.

\section{Acknowledgements}

I wish to thank Erik Koelink for his enlightening discussions and introducing me to the world of the Painlevé equations. I am also grateful to Peter Clarkson for his interest and useful links to the literature.

\section{References}

[1] Yablonskii A.I., On rational solutions of the second Painlevé equation, Vesti AN BSSR, Ser. Fiz.-Tech. Nauk (1959), no. 3, 30-35 (in Russian).

[2] Vorob'ev A.P., On the rational solutions of the second Painlevé equations, Differ. Uravn. 1 (1965), 79-81 (in Russian).

[3] Gambier B., Sur les équations différentielles du second ordre et du premier degre dont l'intégrale est á points critiques fixes, Acta Math. 33 (1909), 1-55.

[4] Lukashevich N.A., The second Painlevé equation, Differ. Uravn. 7 (1971), 1124-1125 (in Russian).

[5] Noumi M., Painlevé equations through symmetry, Translations of Mathematical Monographs, Vol. 223, American Mathematical Society, Providence, RI, 2004.

[6] Airault H., McKean H.P., Moser J., Rational and elliptic solutions of the Korteweg-de Vries equation and related many-body problems, Pure Appl. Math. 30 (1977), 95-148. 
[7] Clarkson P.A., Rational solutions of the Boussinesq equation, Anal. Appl. (Singap.) 6 (2008), 349-369.

[8] Clarkson P.A., Mansfield E.L., The second Painlevé equation, its hierarchy and associated special polynomials, Nonlinearity 16 (2003), R1-R26.

[9] Taneda M., Remarks on the Yablonskii-Vorob'ev polynomials, Nagoya Math. J. 159 (2000), 87-111.

[10] Fukutani S., Okamoto K., Umemura H., Special polynomials and the Hirota bilinear relations of the second and the fourth Painlevé equations, Nagoya Math. J. 159 (2000), 179-200.

[11] Kaneko M., Ochiai H., On coefficients of Yablonskii-Vorob'ev polynomials, J. Math. Soc. Japan 55 (2003), 985-993, math.QA/0205178.

[12] Kametaka Y., On the irreducibility conjecture based on computer calculation for Yablonskii-Vorob'ev polynomials which give a rational solution of the Toda equation of Painlevé-II type, Japan J. Appl. Math. 2 (1985), 241-246.

[13] Kudryashov N.A., Demina M.V., Relations between zeros of special polynomials associated with the Painlevé equations, Phys. Lett. A 368 (2007), 227-234, nlin.SI/0610058. 